\title{
CT Texture Analysis: Defining and Integrating New Biomarkers for Advanced Oncologic Imaging in Precision Medicine: A Comment on "CT Texture Analysis Potentially Predicts Local Failure in Head and Neck Squamous Cell Carcinoma Treated with Chemoradiotherapy"
}

$\mathbf{T}$ he remarkable article by Kuno et $\mathrm{al}^{1}$ in this issue of the American Journal of Neuroradiology raises several questions: Can texture analysis provide reliable biomarkers to predict treatment success in head and neck squamous cell carcinoma (HNSCC)? If so, will the role of the expert clinical radiologist who visually recognizes and interprets image patterns in combination with the clinical impression soon be obsolete and replaced by an increasingly ubiquitous and cheap computing infrastructure for mathematic image analysis, or will radiologists play an even more important role in the future by integrating these new biomarkers for treatment response with their expert knowledge?

In their well-designed study, Kuno et $\mathrm{al}^{1}$ evaluated the performance of pretreatment contrast-enhanced CT texture analysis for the prediction of treatment failure in primary HNSCC treated with chemoradiotherapy. An experienced neuroradiologist, who was blinded to patient history/outcome, contoured the primary tumors manually. An in-house-developed Matlab-based (MathWorks, Natick, Massachusetts) texture analysis program was then used to measure 42 features from each segmented tumor volume. The authors found that 3 histogram features (geometric mean, harmonic mean, and fourth moment) and 4 gray-level run-length features (short-run emphasis, gray-level nonuniformity, run-length nonuniformity, and short-run low graylevel emphasis) were significant predictors of outcome after adjusting for clinical variables, including smoking history, human papillomavirus (HPV) status, T-stage, and tumor volume. ${ }^{1}$

The concept of predicting treatment response based on pretreatment imaging features of HNSCC emerged about 2 decades ago and may be seen as one of the first steps in the development of personalized medicine. During past years, this concept has undergone continuous evolution. The first publications focused on the impact of gross tumor volume on radiation therapy response. In supraglottic HNSCC, preradiotherapy CT-based tumor volume obtained by manual contouring allowed stratification of patients into groups with likely and less likely local control. ${ }^{2}$ Although volume-based prediction of tumor response may be considered an important landmark, radiosensitivity may be influenced by not only volume but also heterogeneity of tumor tissue. Further studies found that glottic HNSCC was better controlled with radiation therapy when cartilage showed a normal or high T2 signal than an intermediate T2 signal. ${ }^{3}$ These observations were later explained by studies correlating preoperative MR imaging with histology, which revealed that a high T2 signal intensity corresponded to inflammation, whereas intermediate T2 signal corresponded to neoplastic cartilage invasion. ${ }^{4}$

Additional imaging biomarkers emerged, such as apparent diffusion coefficient, dynamic contrast-enhanced MR imaging/ CT-derived perfusion parameters, as well as standardized uptake value (SUV) and metabolic tumor volume based on PET. In parallel, researchers evaluated the histologic underpinning of these markers, demonstrating a direct correlation between increased ADC values and high stromal content in HNSCC. ${ }^{5}$ Because high stromal content, low cellularity, and micronecrosis are associated with radioresistance, we now have a possible explanation for the observed poor outcome of patients with high pretreatment ADCs.

As a next step, combined multiparametric approaches then emerged as the new tools for predicting treatment response. In oropharyngeal/hypopharyngeal HNSCC, the combination of large tumor volume and high ADC predicted a higher likelihood of postradiotherapy local recurrence. ${ }^{6}$ Likewise, the combination of ADC and perfusion maps could separate HNSCC responders from nonresponders to chemoradiation, ${ }^{7}$ and more recently, in patients with high maximum SUV, high minimum ADC could identify the patients with the worst prognosis. ${ }^{8}$ The complementary information provided by multiparametric imaging is now increasingly allowing us to reveal the complexity of intra- and intertumor heterogeneity in vivo, and slowly the pieces of a great puzzle are beginning to come together.

Although hailed as a revolution, texture analysis to assess tumor heterogeneity is only the next logical step for predicting treatment response. Image texture can be defined as the spatial variation in pixel intensity levels or patterns, some of which are not perceived by the human eye. The great advantage of this postprocessing tool is that it can be retrospectively applied to data acquired during routine imaging. Assessment of image texture can be done with statistical methods, model-based methods, or transform-based models. ${ }^{9}$ Most publications on texture analysis in oncology are based on statistical methods, which include first-order 
textural features (histograms of pixel-intensity levels based on average pixel value), second-order textural features (gray-level co-occurrence matrices based on the relationship between 2 pixels), and higher-order features based on the relationship between $>2$ pixels. Higher order textural parameters include neighborhood gray tone difference matrices and run-length matrices. While first-order statistical methods do not convey spatial information, second-order and higher-order statistical methods do. Nevertheless, histogram analysis is more intuitive and thus more easily understood by radiologists, whereas second-order and higher-order textural features are more abstract concepts.

Due to its versatility, texture analysis of CT/MR imaging has been recently investigated in several oncologic fields, including assessment of the HPV status in HNSCC ${ }^{10,11}$ or survival of patients with advanced HNSCC treated with induction chemotherapy. ${ }^{12}$ The article by Kuno et al $^{1}$ fits in this timely area of research and demonstrates some remarkable findings. CT, which is readily available in many institutions, can be used to extract meaningful texture information, allowing prediction of treatment outcome irrespective of scanner type and section thickness or the use of iterative reconstruction.

From a general scientific point of view, several methodologic challenges must still be overcome before texture analysis will be ready for routine clinical use in head and neck $(\mathrm{HN})$ oncology. First, the technical platforms for texture analysis are not yet standardized, and even minor differences in equipment, acquisition protocols, or the presence of artifacts may significantly affect texture features, thus questioning whether the obtained results can be reproduced by another technical platform. Ideally, scientific studies correlating texture-based biomarkers with treatment outcome should, therefore, be conducted on the same scanner, with the same protocol, and in a well-defined homogeneous subgroup of patients. This problem is generally inherent in quantitative image analysis and is currently being addressed by international research alliances such as the Quantitative Imaging Biomarker Alliance and the European Imaging Biomarker Alliance. Second, segmentation of $\mathrm{HN}$ tumors, a key ingredient for any meaningful texture analysis, remains a time-consuming procedure, which must be done manually and based on visual assessment by an experienced radiologist. In view of the complex morphology of HN tumors, reliable digital automatic segmentation tools based on artificial intelligence may be difficult to develop for this particular purpose. Third, we must agree on a standard method for manual segmentation to make data comparable and reproducible. Should we contour only the most representative tumor section or rather include the entire tumor volume in the analysis? Should we include or exclude necrotic portions or ulcerated tumor parts from our analysis? Such questions need to be answered to avoid noise due to inconsistent data analysis and allow a meaningful correlation of texture features with treatment outcome. Fourth, the scarcity of histopathologic, functional, or metabolic correlates often implies that statistical power cannot be obtained unless data can be shared among institutions.

Finally, the question remains about how far we must go to understand the underlying biologic mechanisms influencing texture analysis, such as cellularity, hypoxia, or angiogenesis. Some may argue that it is sufficient to provide biomarkers with proved correlation between treatment and outcome, whereas others may insist that true scientific progress will not be possible without a real understanding of the biologic correlates of surrogate imaging biomarkers.

Texture analysis is now entering the area of personalized medicine, accompanied by sensationalistic comments in the lay press and a media hype announcing a new revolution in oncologic research. There is, indeed, little doubt that the possibility of developing biomarker-based texture analysis is promising for the progress of oncologic imaging, though many scientific questions still need to be answered. The work of Kuno et $\mathrm{al}^{1}$ is a significant contribution and takes us a step ahead. From a clinical point of view, there is still some way to go before texture analysis can be effectively implemented for the benefit of our patients. Those of us who are actively taking part in multidisciplinary $\mathrm{HN}$ tumor boards are fully aware that the not-so-well-quantifiable clinicalradiologic impression will continue to play an important role in multidisciplinary therapeutic decision-making.

The upcoming challenge will consist of integrating the information of biomarkers derived from multiparametric texture analysis with the more pragmatic image interpretation of the experienced clinical radiologist. The goal of clinical imaging remains to reliably provide a positive impact on the treatment and outcome of our patients. I personally believe that this may be done best by integrating the new exciting biomarkers gradually as soon as they have been proved to be scientifically reproducible, following Theodore Roosevelt's advice, "Keep your eyes on the stars and keep your feet on the ground."

\section{REFERENCES}

1. Kuno H, Qureshi MM, Chapman MN, et al. CT texture analysis potentially predicts local failure in head and neck squamous cell carcinoma treated with chemoradiotherapy. AJNR Am J Neuroradiol 2017 Oct 12. [Epub ahead of print] CrossRef

2. Mancuso AA, Mukherji SK, Schmalfuss I, et al. Preradiotherapy computed tomography as a predictor of local control in supraglottic carcinoma. J Clin Oncol 1999;17:631-37 CrossRef Medline

3. Ljumanovic R, Langendijk JA, van Wattingen M, et al. MR imaging predictors of local control of glottic squamous cell carcinoma treated with radiation alone. Radiology 2007;244:205-12 CrossRef Medline

4. Becker M, Zbären P, Casselman JW, et al. Neoplastic invasion of laryngeal cartilage: reassessment of criteria for diagnosis at MR imaging. Radiology 2008;249:551-59 CrossRef Medline

5. Driessen JP, Caldas-Magalhaes J, Janssen LM, et al. Diffusionweighted MR imaging in laryngeal and hypopharyngeal carcinoma: association between apparent diffusion coefficient and histologic findings. Radiology 2014;272:456-63 CrossRef Medline

6. Ohnishi K, Shioyama Y, Hatakenaka M, et al. Prediction of local failures with a combination of pretreatment tumor volume and apparent diffusion coefficient in patients treated with definitive radiotherapy for hypopharyngeal or oropharyngeal squamous cell carcinoma. J Radiat Res 2011;52:522-30 CrossRef Medline

7. Chawla S, Kim S, Dougherty L, et al. Pretreatment diffusionweighted and dynamic contrast-enhanced MRI for prediction of local treatment response in squamous cell carcinomas of the head and neck. AJR Am J Roentgenol 2013;200:35-43 CrossRef Medline

8. Preda L, Conte G, Bonello L, et al. Combining standardized uptake value of FDG-PET and apparent diffusion coefficient of DW-MRI improves risk stratification in head and neck squamous cell carcinoma. Eur Radiol 2016;26:4432-41 CrossRef Medline

9. Alobaidli S, McQuaid S, South C, et al. The role of texture analysis in 
imaging as an outcome predictor and potential tool in radiotherapy treatment planning. Br J Radiol 2014;87:20140369 CrossRef Medline

10. Buch K, Fujita A, Li B, et al. Using texture analysis to determine human papillomavirus status of oropharyngeal squamous cell carcinomas on CT. AJNR Am J Neuroradiol 2015;36:1343-48 CrossRef Medline

11. de Perrot T, Lenoir V, Domingo Ayllón M, et al. Apparent diffusion coefficient histograms of human papillomavirus-positive and human papillomavirus-negative head and neck squamous cell carcinoma: assessment of tumor heterogeneity and comparison with histopathology. AJNR Am J Neuroradiol 2017 Sep 14. [Epub ahead of print] CrossRef Medline
12. Zhang $\mathrm{H}$, Graham $\mathrm{CM}$, Elci $\mathrm{O}$, et al. Locally advanced squamous cell carcinoma of the head and neck: CT texture and histogram analysis allow independent prediction of overall survival in patients treated with induction chemotherapy. Radiology 2013;269:801-09 CrossRef Medline

(D) Mecker Division of Radiology Department of Imaging and Medical Informatics Geneva University Hospital Geneva, Switzerland 